\title{
INTRODUCING MULTIDISCIPLINARY DESIGN OPTIMIZATION TO GRADUATE LEVEL ENGINEERING STUDENTS
}

\author{
Papageorgiou, Athanasios; \\ Wiberg, Anton; \\ Persson, Johan \\ Linköping University
}

\begin{abstract}
Multidisciplinary Design Optimization (MDO) is a method that has shown many promising results in the development of complex engineered products. To this date, research on MDO has been extensive, but at the same time, very few publications have addressed the aspect of how it can be taught to students and young professionals. In this light, this paper aims to present the experiences of the authors in respect to the development and management of an MDO course at Linköping University. First, this work will describe the authors' teaching approaches, and in particular, it will present the various educational activities that have been considered over the years as well as the lessons learnt. Secondly, this work will attempt to investigate how students perceive a set of common MDO concepts, and more specifically, it will present an analysis based on the results of two surveys that took place in 2016 and 2020, respectively. Given the above foundation, this paper will try to establish guidelines regarding the activities which are suitable for teaching each concept, while finally, it will also touch upon the challenges as well as the solutions for adjusting an MDO course to a distance learning mode.
\end{abstract}

Keywords: Education, Design methodology, Optimisation

\section{Contact:}

Papageorgiou, Athanasios

Linköping University

Management and Engineering

Sweden

athanasios.papageorgiou@liu.se

Cite this article: Papageorgiou, A., Wiberg, A., Persson, J. (2021) 'Introducing Multidisciplinary Design Optimization to Graduate Level Engineering Students', in Proceedings of the International Conference on Engineering Design (ICED21), Gothenburg, Sweden, 16-20 August 2021. DOI:10.1017/pds.2021.453 


\section{INTRODUCTION}

Over the last decades, the constant advancements in technology together with the increasing customer demands have pushed the industry to reach new frontiers by developing even more complex systems. This new trend has generated further technical and managerial challenges for every product development team, and as a result, engineers and companies are nowadays compelled to enhance their practices by following more efficient approaches and considering more advanced design tools. To this end, a design space exploration method that has been frequently implemented in the development process of complex engineered products is Multidisciplinary Design Optimization (MDO). The primary aim of MDO is to help engineers unveil the system's emergent behaviour by capturing its underlying dependencies, while at a secondary level, a further goal is to reveal the best-suited designs by using numerical optimization. Over the years, the potential and benefits of MDO have been successfully verified in both an academic as well as an industrial setting, and overall, it has been shown that it can be adapted to various stages of the development process and to various complex engineered products.

Although MDO is nowadays considered a well-established method, a report from a workshop where various experts from the industry and academia were invited revealed that there is still a lot of margin for improvement (Simpson and Martins, 2011). Here, it was identified that four out of the five directions for future work were related to the technical aspects of the method, however, the fifth point that the panel of experts identified as critical was in respect to education. Consequently, it was argued that MDO needs to be integrated creatively in the engineering curriculum, and more specifically, it was claimed that universities need to foster a "state of mind" that will in turn allow the alumni to bring this method out of the classroom and into the corporate culture. Along the same lines are the conclusions of an earlier review on MDO practices which was performed by a broad group of well-established individuals in the field (Agte et al., 2010). In this work, the authors identified that there is an absence of MDO education both at the university as well as in the industry, and they argued that the main problem with the existing courses is that they fail to attract engineers because they focus on mathematics. Apart from that, it was further mentioned that the MDO education comes too late in the curriculum and that there are very few relevant textbooks, while finally, it was observed that there is the lack of educational toolboxes which can help students see the effects of MDO on real-life system engineering cases.

In light of the above, the aim of this paper is to present the experiences of the authors on the development of an MDO course at Linköping University. At a primary level, the goal herein is to give answers to the questions "How should MDO be taught in higher education?", and "Which learning activities can promote a better understanding of MDO?". Consequently, this work will initially describe the authors' teaching approaches, and in particular, it will present in detail the various educational activities that have been considered over the years as well as the lessons learnt. In addition to the above, a further objective of this paper is to provide answers to the questions "Which MDO concepts are difficult to grasp?", and "What should be added/discarded?". To be able to answer those, this work will subsequently bring forward the results of two surveys that took place in 2016 and 2020, and more specifically, it will present an analysis which will attempt to shed some light into the students' understanding of various MDO topics as well as their views on the effectiveness of the chosen teaching methods. Finally, this paper will touch briefly upon on the issue of distance learning which was enforced in 2020 because of the COVID19 restrictions, and to this end, it will also present the authors' latest adjustments to the structure of the course and how those were ultimately received by the students.

Overall, this paper is divided into 6 chapters, with the introduction being the first, followed by the frame of reference. The next chapter in line is the description of the course and then comes the analysis of the student survey findings. Finally, the paper summarizes with a short discussion and conclusions.

\section{FRAME OF REFERENCE}

\subsection{Teaching Experiences}

One indicative example of how MDO was included in the under- and postgraduate engineering programmes of Virginia Tech was presented by Mason et al. (1995). In this work, the authors describe their experiences in respect to the development of an MDO course which was based on an aircraft design project, while in addition to this, they discuss the results from a survey which they conducted in order to investigate the students' perception of the taught concepts. According to the authors, the combination of a multi-fidelity multidisciplinary project-based approach can promote creative thinking and lead to a 
positive learning outcome, however, they also identified that the experience can be further enhanced if the students are given close-ended tasks which can familiarize them with specific topics. Nevertheless, the authors identified several challenges which were coupled to the diverse background of the students, and more specifically, they observed problems in the understanding of the disciplinary interactions and the use of tools for developing the necessary disciplinary models.

Two further examples of how MDO was addressed at Stanford University and Georgia Tech were presented respectively by Gerber and Flager (2011) as well as Nicknam et al. (2013). In both cases, the authors start by giving an overview of the potential tasks and assignments which can be linked to the concurrent architectural-civil design of buildings through CAD/CAE tools, while finally, they conclude by indicating a list of related educational activities which can be used to give a broader view of MDO and its benefits within product development. According to Gerber and Flager (2011), their project-based learning approach enabled students to see the benefits of MDO compared to a traditional iterative design process, but nevertheless, they also noticed that the students struggled in the initial stages of the project with model creation as well as model parametrization. Similarly, Nicknam et al. (2013) observed that students appreciated working as domain experts in multidisciplinary teams as well as dealing with actual industry problems, however, they also identified that such an approach raises challenges in respect to the integration of different tools, the formulation of the right design problem, and the ability to explore and in turn visualize the design space in a meaningful way.

Lastly, there are the two examples on the topic of educational toolboxes for supporting MDO teaching, and more specifically, the aerostructural analysis and optimization tool based on NASA's OpenMDAO framework (Jasa et al., 2018) as well as the AGILE aircraft design capabilities paradigm of DLR (Della Vecchia et al., 2018). Two MDO courses at Toulouse University and Michigan University are presented to illustrate the benefits of the former, whereas the application of the latter toolbox is introduced in the context of the AGILE academy which in total includes 36 international universities. The main benefit of the above toolboxes is their ability to produce simple MDO problems at variable levels of fidelity, while in addition to this, their modular nature allows the user to connect external tools, to work with various aircraft cases, and to experiment with different problem formulations.

\subsection{Research Foundation}

Since the emergence of the first MDO studies almost 35 years ago, the field has expanded rapidly in many different directions, and nowadays, there are several research areas which are focusing on various aspects of MDO (Simpson and Martins, 2011). The structure of the presented MDO course at Linköping University has been based on the clustering which was proposed in the review of Papageorgiou et al. (2018). According to this taxonomy, the MDO conceptual components can be divided into three clusters of activities which start with the definition of the optimization requirements, move to the development of the framework, and conclude with the data management (See Figure 1).

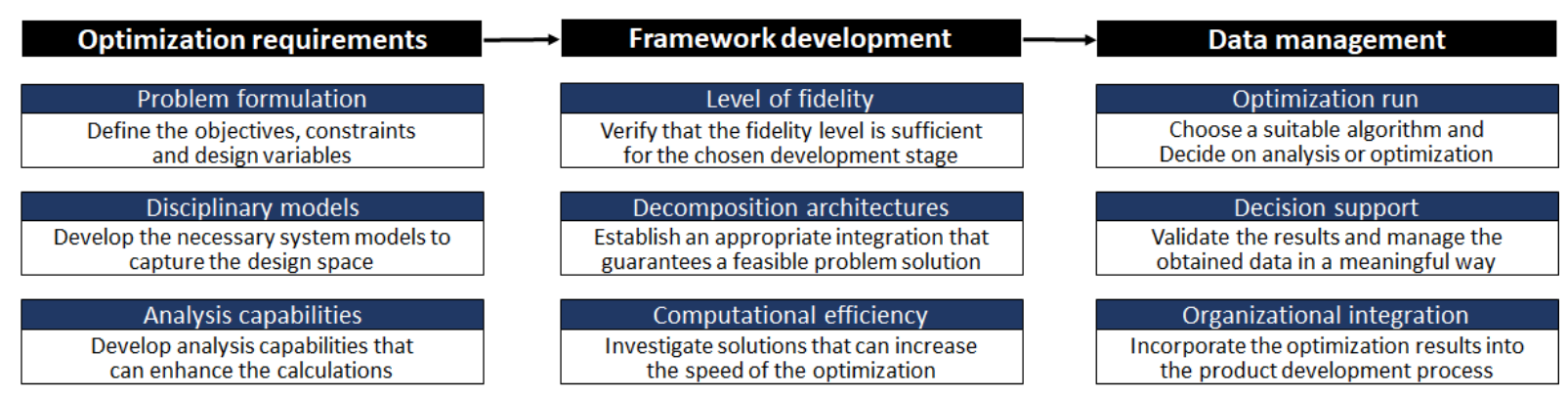

Figure 1. The three MDO clusters according to Papageorgiou et al. (2018)

The first cluster of activities is about translating the requirements, and in general, this includes the mathematical formulation of the optimization problem as well as the development of the modelling and analysis capabilities. According to Simpson and Martins (2011), the former point is about translating the complex customer needs into quantifiable design attributes, and more specifically, this process should result in a suitable set of objectives, variables, and constraints which can be used to explore the trade-offs among the various design aspects of complex systems. In addition to this, there is a need to represent the system, and therefore, a further consideration for a successful MDO implementation is to develop the right set of models and enable the required analysis capabilities (Agte et al., 2010). Thus, the nature and 
focus of each study is what defines the type of models which will be used for capturing the emergent behaviour of the system, while overall, the computational fidelity of the tools and the extent of the analysis capabilities will be decided based on the stage and goals of the development process that MDO aims to enhance (Giesing and Barthelemy, 1998).

The second cluster of activities is about the development of the framework which will enable the study of the complete system and in turn allow for an optimization of the design. At this stage, the focus is on the integration of the system models on a common platform which is typically expressed and guided by means of a suitable decomposition architecture. The most common function of MDO architectures is to define how the system models are coupled and how the optimization problem will be solved. However, architectures can also be used to achieve solutions with specific algorithms and to take advantage of parallel computing capabilities (Martins and Lambe, 2013). Apart from the above, this stage is also about aligning the level of computational fidelity with the goals of the MDO study, and here, the two identified solutions are to either follow a multi-fidelity approach or to develop approximation models. In the former case, the faster low-fidelity methods are used initially to explore the entire design space, and then the slower high-fidelity models are engaged over a smaller area (Agte et al., 2010). In the latter case, the computationally expensive models are analysed at a small set of design points, and then the inputs and outputs are correlated be means of machine learning algorithms in order to create a much faster, but also less accurate, surrogate- or metamodel (Viana et al., 2014).

The third and final cluster of activities is about running an optimization and interpreting the results in a meaningful way. Here, it has been argued that raw optimization data does not hold any significance for the decision makers unless it is presented in the right way, and thus, a frequently identified factor for promoting the organizational integration of the method is to complement MDO with proper visualization techniques (Giesing and Barthelemy, 1998). Along the same lines, Simpson and Martins (2011) argue that MDO is a tool to help people make better decisions but it will never replace human intuition, and in this respect, practitioners should remember that the results must always be validated to ensure both the feasibility as well as the realism of the suggested solutions.

\section{COURSE STRUCTURE}

\subsection{Overview and Evolution}

The aim of this section is to present an overview of the course's structure, starting from its introduction in 2014 and reaching to the time of writing this paper in 2020. A shared characteristic in all years that the course has been running is the consideration of a project-based learning approach, however, over the years different methods have been followed in terms of teaching as well as examination. A summary of the most important contextual and organizational aspects of the course per academic year can be found in Table 1, while a more detailed description is provided in the following sections.

Table 1. Overview of the course's organization per academic year

\begin{tabular}{|c|c|c|c|c|c|c|c|}
\hline Year & 2014 & 2015 & 2016 & 2017 & 2018 & 2019 & 2020 \\
\hline Examination & Report & \multicolumn{2}{|c|}{ Presentation } & Presentation + Individual Exercise & Presentation \\
\hline Project & Common & Free choice & Common & 2 Options & 3 Options & 2 Options \\
\hline Models & Not given & Not Given & Some of them given, and some of them had to be developed \\
\hline Groups & \multicolumn{2}{|c|}{ 5 Students } & \multicolumn{5}{|c|}{ 2-3 Students } \\
\hline Grading & Expert judgement & \multicolumn{5}{|c|}{ According to a list of milestones and tasks } \\
\hline Literature & Mandatory & \multicolumn{7}{|c|}{ Provided as additional support } \\
\hline Tutorials & Mandatory & Recommended but not mandatory to complete them & Mini project \\
\hline Lectures & Intro + 1 & Intro + 1 & Intro + 5 & Intro +6 & Intro + 7 \\
\hline Teaching & \multicolumn{7}{|c|}{ Group mentors + Lab assistants } \\
\hline
\end{tabular}

\subsection{Project Work}

In 2014 the examination project was based on a common task that the students had to complete by following specific instructions in the form of tutorials. First, the students were asked to develop various models that represented several design aspects of an underwater (tidal) turbine system, and then they were asked to integrate them in a common framework in order to perform MDO. Here, the focus was to illustrate the benefits of MDO through high-fidelity models, and to this end, the project considered the 
product geometry (CAD), flow (CFD) as well as structural (FEM) analyses, and power calculations (Dynamic Simulation). In addition to this, the learning objectives briefly covered the topic of developing and using metamodels, while finally the students were asked to do a literature review and in turn compare knowledge from other studies against their own MDO results.

In 2015 the format changed to a free-choice project where the students had to first find a suitable product and then set up an MDO study by themselves. Compared to the previous year, the models were neither decided in advanced nor given, and therefore, it was up to the student groups to develop them according to the requirements of each design case. Here, the students could work with the same high-fidelity tools that were available for the 2014 class, but in addition to this, it was also possible to develop their own case-specific calculations in a mathematical software of their choice. To guide the projects in the right direction, a list of potential research topics was provided, and the students were asked to address several of them by using their frameworks. Some of the suggestions were the development of metamodels, the study of decomposition architectures, and the investigation of various problem formulations. In total, 8 different projects were undertaken, and those were a race car spoiler, a helicopter blade, a toy airplane, a quadcopter, a lifting crane, a submarine, a wind turbine, and a golf club.

In 2016 the course introduced to a common project for all the students. The main reason was the lack of available modelling solutions for supporting the free-choice projects, but it was also identified that the current tools made the students spend a high amount of time on tackling integration issues. The proposed project was based on a commercial aviation aircraft which considered several models at variable levels of computational fidelity. All the aeronautical models were given to the students since most of them did not have a relevant background, and therefore, the focus here was shifted to the development of a generic structural model of the fuselage by using a FEM tool. Apart from modelling, various obligatory tasks were also added to ensure that all the aspects of MDO are understood. For grade 3 (lowest) the project required an analysis of the results and a sensitivity study; for grade 4 (average) the requirement was to investigate the use of metamodels; and lastly, for grade 5 (highest) the students were asked to explore the feasibility of different decomposition architectures.

The course followed the same pattern as above between 2017 and 2020, but with the difference that instead of one, several projects were offered to the students. Overall, the grading scale remained the same as in 2016, however, it should be noted that some occasional changes had to be often implemented in order to align the level of difficulty. As expected, different models were given to students according to the type of project, but in general, all projects included a high-fidelity aspect which was in all cases expressed by means of some sort of structural analysis model. In 2017 there were two available options (supersonic passenger aircraft, factory industrial stairs); in 2018 three (bridge construction, aircraft wing, passenger car); in 2019 three (wind turbine, additive manufacturing part, passenger car); and finally, in 2020 two (space exploration radar, passenger car).

\subsection{Examination Activities}

In all the years except the first, the examination of the students and the grading of the project has been primarily based on a final presentation. The goal is to present the details of the project work (model development, framework integration, etc.), while at the same time, the students are also given the opportunity to motivate their choices and discuss the obtained results. A 15-minute timeslot is typically allocated for each presentation, and then the rest of the time is reserved for questions and comments. This is typically sufficient, because by the time of the final presentation the mentors of each group have already a good picture of the students' work quality and level of efforts.

An examination element that has been used since 2017 is the so-called "individual exercise". This is essentially a computer-based exam where the students are asked to conduct an MDO on a small-scale problem. Here, the students are given a set of simple equations which they must use to develop models, and then, they are asked to setup a framework, run an optimization, and discuss the obtained results. The reason is to ensure that all students have the required knowledge and that no one was a "passenger" in the group. Due to the distance learning mode, in 2020 the individual exercise was temporarily replaced by individual questions which were posed to the students after the end of the presentation.

The "mini project" is a course element that was introduced in 2020 in order to help teachers identify the potential gaps of each group early in the course. A similar format as in the final presentation was followed, but the main difference was that the students discussed the results which they obtained from the tutorials. Overall, the students received feedback on their technical work as well as their 
understanding of MDO concepts, but most importantly, this was also an opportunity to see what is really asked by this course and which directions to follow in order to get there.

\subsection{Learning Activities}

Apart from the project work, the course curriculum includes several supplementary learning activities which aim to enhance the students' knowledge on MDO topics. From the early course evaluations, it was identified that most students were missing a theoretical foundation, and hence, it was decided to address that through lectures. Over the years, several topics have been added to the list of offered lectures, and an overview of the changes and content is given in Table 2. Accordingly, several literature sources are provided every year, however, those are not mandatory to read (except in 2014), but they are rather given as "extra" material for those who want to delve deeper into a specific area.

Table 2. The content of lectures per academic year

\begin{tabular}{|c|c|c|c|}
\hline Year & Lecture & Year & Lecture \\
\hline $14-20$ & Course presentation / Intro to MDO & $16-20$ & Decomposition architectures \\
\hline 14 & Guest lecture on aerodynamic modeling & $16-18$ & Roles and collaboration in MDO \\
\hline $15-20$ & Development of metamodels & $17-20$ & Post-optimal analysis and visualization \\
\hline $16-20$ & General optimization knowledge & $19-20$ & Guidelines for best MDO practice \\
\hline $16-20$ & Research/industrial MDO examples & $19-20$ & Model fidelity / Development process \\
\hline
\end{tabular}

A learning activity that has shown many promising results in terms of teaching both the theoretical as well as the practical aspects of MDO is the use of tutorials. Over the years, there have been three versions of the tutorials, while in addition to them, some extra material from other courses has also been provided in order to cover gaps in the prerequisite knowledge (e.g., setting up a FEM analysis). In 20142015 the tutorials focus primarily on model development by using an underwater turbine as an example, while at a secondary level, they covered the topics of metamodels and integration/optimization. Between 2016 and 2019 the MDO topics were clustered into three main tutorials which used the example of a human-powered aircraft, and more specifically, they covered the basic integration optimization strategies; the development of metamodels; and the implementation of different decomposition architectures. In 2020 the overall format of the tutorials was the same, however, a different example was used (wind turbine), and some further explanations were added according to identified gaps.

In the year 2020 several adjustments had to be made to the course in order to ensure that it could meet the requirements for distance learning. The course material was distributed by online sharing platforms as it was previously done; communication with the students was now through emails, text messages, or video calls; and finally, lectures were given exclusively online in either a "live" form by using meeting tools or in a pre-recorded "video" form. Here, the main challenge proved to be the access to the university computer labs which had the necessary software tools for completing the project. To this end, a remote desktop solution allowed the students to work on the dedicated computers, whereas as far as technical support is concerned, the students were also given the opportunity to share their screen or even give full control of their computers to the assistants/mentors.

\section{STUDENT EVALUATION}

The course quality and content were measured in two different kind of surveys. The first is a survey by the university which measures the quality of the course each year. This survey is emailed to the students at the end of the course and it is answered online. The overall course quality is judged on a scale from one to five. The average course score and the percentage of the course participants that answered the survey for each year is presented in Figure 2.

As a compliment to the standard survey performed by the university, a more in-depth survey to analyse the course content was performed at the end of the course in 2016. The same survey was also performed in 2020. In 2016 the survey was performed with pen and paper in conjunction with the presentations. 24 out of the 30 course participants answered the survey. In 2020, the survey was emailed to the students and performed digitally. Two reminders to complete the survey were emailed to the students. 13 out of the 54 students who took the course answered the survey.

The survey included free text answers where the students answered questions related to which topics in the course they judged as most difficult to understand, most important etc. Keywords related to MDO 
concepts presented in the course were, by the authors, identified in the answers and group the answers into different categories. The distribution of answers belonging to the different categories is presented in Figure 3. In addition to this, the respondents were also asked to evaluate (on a scale from one to five) the different MDO concepts, based on how difficult it was to understand and how useful to know they were according to the participants. The results of this evaluation are presented in Figure 4.

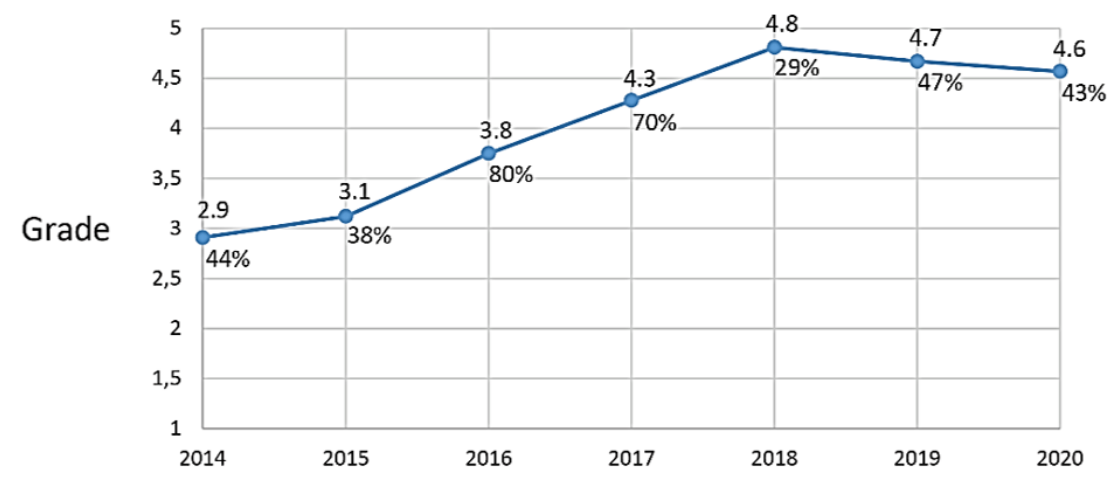

Figure 2. The average course score per academic year

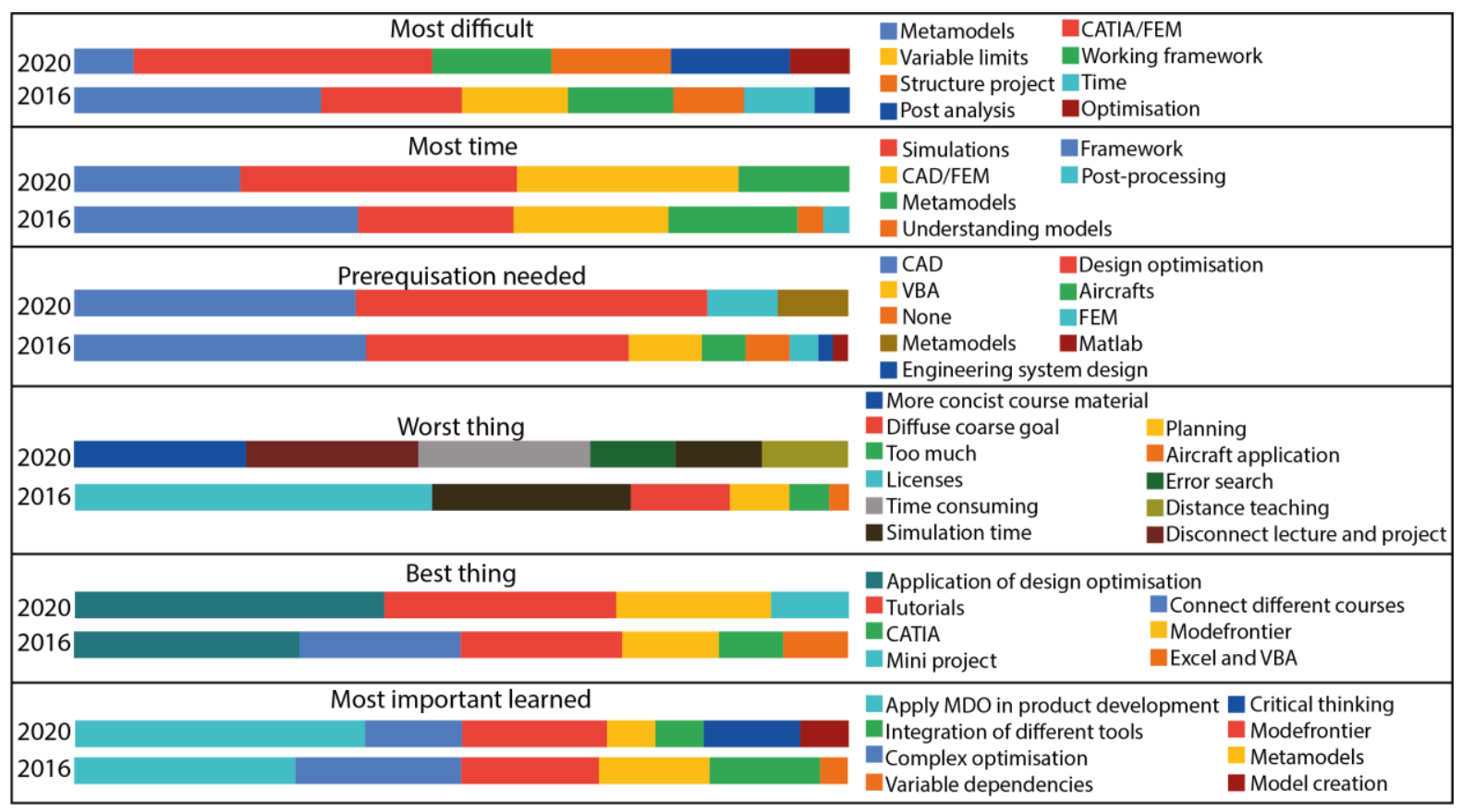

Figure 3. The survey answers to the free text questions

The answers of the two last questions, considering the "most important learned" and "best thing" with the course, show that students appreciate the opportunity to implement design optimisation strategies to real world problems. Earlier optimisation courses, which most students have participated in, are focused on basic optimisation concepts, approaches, and algorithms. The goal of this course, which the answers confirmed, is to teach how to implement this in real life scenarios. The course also focuses on how MDO can be implemented in the general engineering design process. As seen in Figure 4, most of the MDO concepts get a high score regarding how useful to know they are.

Although the students should have participated in several CAD courses with everything from basic to advanced CAD, both the question of "most time spent" and "most difficult to solve", show that CAD modelling for MDO purpose is complex. The requirement that the CAD model should be flexible and robust but also possible to use in other CAE applications adds a complexity to the model which has not been taught in earlier courses. This clearly shows that modelling for MDO is an important subject which traditionally is not taught in modelling courses.

Both in 2016 and 2020 the students judged that one of the activities which took most of the time was simulation. As simulation time, and cost, is also one of the challenges with MDO this is a good lesson although the students do not seem to appreciate it. Some actions have been taken in order to make it 
easier for the students. Data of computer expensive models have been given, and in 2020 the students were given access to simulation computers which could be used full time. In 2016 they could only use the computers in the computer halls (which were booked for other courses during certain hours).

From a course perspective it can be observed that the mini-project is mentioned as one of the best things of the course in 2020. This is an indication that the mini-project was appreciated by the students. If the mini-project improved the overall learning of the subject is however not clear. Both in 2016 as well as in 2020 the "worst things" of the course are coupled to practical aspects within the course and not aspects coupled to the topic of MDO itself.

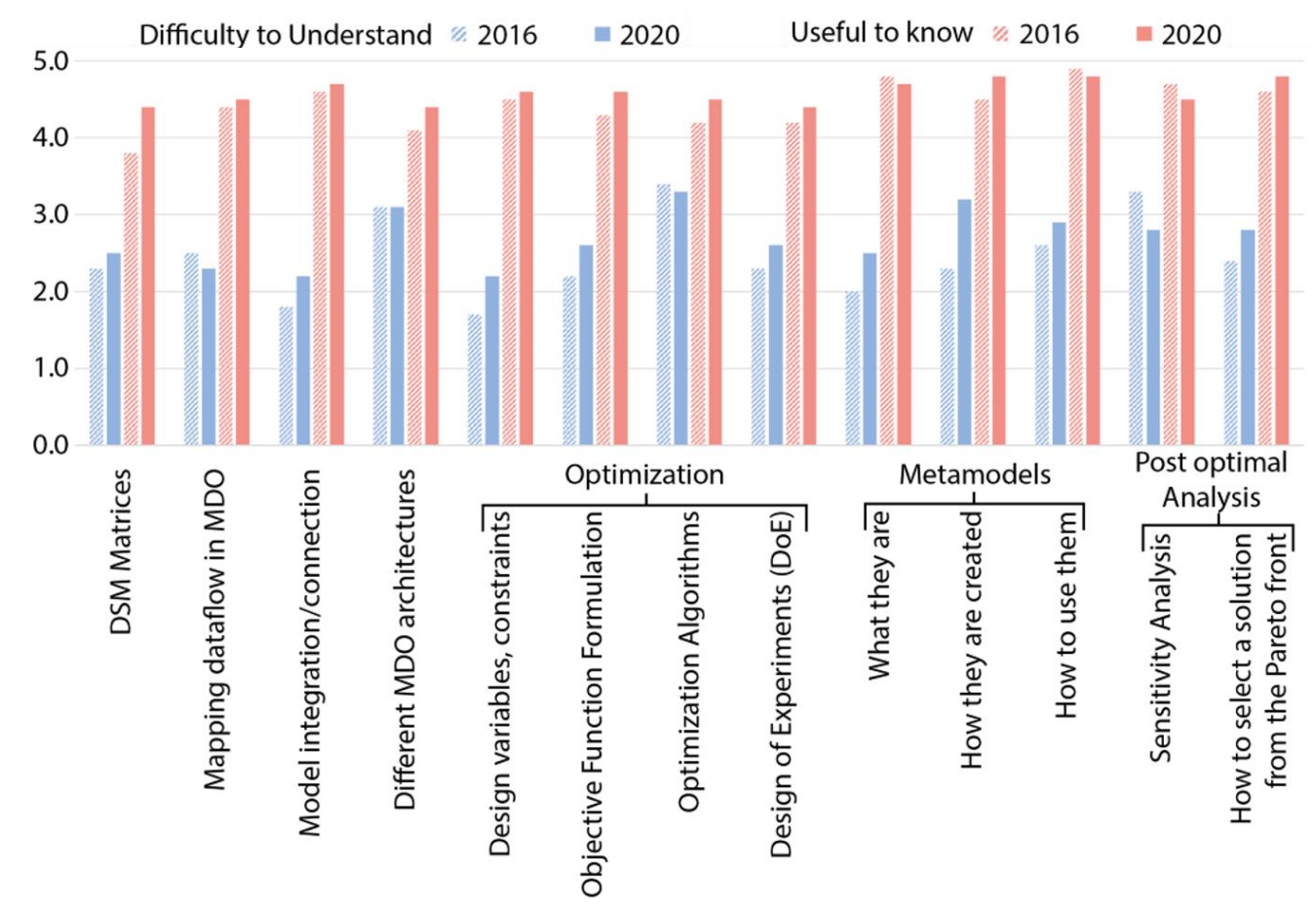

Figure 4. Evaluation of different MDO concepts based on difficulty and importance

Based on what the respondents judge as difficult to understand, four things can be highlighted. The structure of the project and the architecture are difficult, while the tightly coupled subject of variables and constraints as well as the objective formulation are not. For the former, the judged difficulty has increased from 2016 to 2020, which may be coupled to the nature of the project. In 2016 everybody did the same strict project, whereas in 2020 two different more open-ended projects were performed.

The subject which the students ranked as most difficult, both in 2016 and 2020 was the optimisation algorithms. This is a surprise as a central prerequisite is a previous course in design optimisation where all the different types of optimization algorithms are explained. It could be argued that some of the students have not taken or performed well in that prerequisite course, but it could also be possible that they are having difficulties to apply their previous knowledge in this new context.

The decomposition architectures are the second most difficult subject according to the survey. As expected, architectures can be inherently difficult to grasp because they have not been covered in other courses and because one is faced with an increased number of variables and couplings. Nevertheless, this topic is covered by a dedicated lecture as well as a tutorial, while furthermore, the students are only asked to work with one of the simplest architecture types.

Metamodels are the third most difficult subject. It has been observed during teaching that students do not think it is difficult to create meta models but testing and achieving a good accuracy is often difficult. Even though this topic is also covered by a dedicated lecture as well as a tutorial, there seems to be some confusion on what a metamodel is and how it can be used in the context of MDO.

The fourth and last subject which receives a high score of difficulty is post analysis of the result. This area is often neglected by students who do not do anything more apart from selecting the most optimal design or presenting several Pareto optimal solutions. The selection of a design from a Pareto front is a challenge for most students, whereas it has alco been observed that it is generally difficult to analyse and select designs based on the robustness of the solution. 


\section{DISCUSSION}

The analysis of the course evaluation and the student feedback suggests that project-based learning can be a suitable approach for teaching MDO as it has also been shown in the relevant literature (Mason et al., 1995; Gerber and Flager, 2011; Nicknam et al, 2013). This allows the students to see how a typical MDO process looks in the context of a realistic case study, while furthermore, it helps them understand the expected challenges and possible solutions that are coupled to the implementation of the method. Nevertheless, giving the students the freedom to choose their own products and tools could lead to problems of technical nature which in turn can hinder the learning process. Therefore, it is argued here that a moderately "guided" project can enable students to get a better understanding of MDO, since they do not need to put so much effort on its practical implementation which according to the survey is tedious and time-consuming. In principle, this follows the suggestion of Agte et al. (2010) regarding the development of educational "toolboxes", like in the examples of Jasa et al. (2018) and Della Vecchia et al. (2018) for aeronautical applications. To this end, part of the problem formulation and several of the building blocks can be provided in order to guarantee a feasible and timely completion of the project, but at the same time, the margin for decision making should be carefully planned in order to ensure that the student creativity and self-learning opportunities will not be constrained.

In addition to the above, the authors' observations and the student survey revealed that there are often significant difficulties with respect to understanding the various MDO concepts. Therefore, the proposed teaching approach includes also learning activities that aim to help students build a better theoretical foundation and gain an in-depth understanding of the potentials and limitations. This will help foster the development of a "state of mind" which was identified as a critical gap by Simpson and Martins (2011). According to the survey, the use of tutorials as a medium to disseminate knowledge seems to be very effective, and the reason is that there is an obvious application of the theory. Conversely, the lectures do not appear to be appreciated in the context of MDO teaching, and in fact, the challenge appears to be the lack of a direct connection to the project. To tackle the above and given that the use of extra material does not seem to help, two possible solutions would be to either steer the lectures towards more practical matters or to move some of the taught material into a tutorial form.

The experience of the authors with distance learning in 2020 showed that it is possible in the context of a project-based course. This is also supported by the student survey, where only a very small percentage of students viewed this as a challenge. Nevertheless, several issues were identified and need to be further addressed. One issue is that in the traditional lab setting the students get more chances to interact with the teachers, whereas in the distance learning mode the communication is only limited to a narrow list of topics. As a result, the supervisors have fewer chances to get to know each student; they have less knowledge over the group's progress; and most importantly, they do not get the opportunity to talk about the "big picture" behind the course. As expected, it is also more difficult to provide troubleshooting in case of technical problems, and in fact, the "error search" seems to have taken a big proportion of the student's time in 2020. Finally, there is the issue of engaging the students in the learning activities. For reasons that are not clear, only half of the students participated in the online lectures, while interestingly, there were only just a few individuals who saw the pre-recorded lectures.

One possible limitation of the survey is the number of participants. In 2016, the questionnaire was answered by $80 \%$ of the class, whereas in 2020 only $24 \%$ of the students responded. In addition to this, the grades of the students were not considered. If the questions were answered by students with high academic performance, it could mean that the answers do not reflect the actual situation. Furthermore, it is important to consider the skills of the teachers. Several teams of teachers have worked in this course over the years, which suggests that the students have not received the exact same supervision. Lastly, it should be noted that the project difficulty and other exogenous factors could also have influenced the results. For instance, a higher overall course workload, internal group problems, or reduced interactions due to the distance learning mode could have had a negative impact.

Apart from the above, an issue that the authors would also like to raise is the fact that educational teams are typically skilled within certain research areas. This implies that there are limitations when it comes to the taught concepts but also to the possible applications of the method. In this course, the teaching team chose to exclude the topic of uncertainty propagation, because it was considered too complex to cover in addition to the other topics. For the same reasons, the topics of MDO architectures as well as the organizational integration of MDO received a low emphasis when compared to the topics of problem formulation, results postprocessing, and development of metamodels. Accordingly, the team chose to 
focus on certain products, and more specifically, the authors took advantage of material from previous projects in mechanical as well as aeronautical engineering.

Even though a lot of effort has been put into the development of this course, the survey suggests that there is still margin for improvement. Students seem to appreciate the fact that they can work on realistic case studies, and thus, an important aspect for future development is to strive to include more complex products which will in turn engage an even broader audience. To this end, the toolboxes are constantly expanding by either adding entirely new tasks which have been inspired from research projects or by improving the existing ones with more analysis formulation options. Moreover, it would be beneficial to include more higher fidelity analysis capabilities as this is what makes MDO different from the basic mathematical optimization courses. However, this needs to be done in an efficient way that does not overload the students with unnecessary practical tasks and waiting time, and hence, it is also important to make sure that the software tools and the hardware infrastructure can efficiently support the project. Lastly, more efforts are needed in respect to teaching the various MDO concepts as well as including new ones. The survey showed that there are still major difficulties in terms of understanding the theory behind MDO, and thus, the authors continue their exploration of the best teaching method by evaluating further learning activities, mentoring approaches, and examination methods.

\section{CONCLUSIONS}

This work discusses the development of a course on Multidisciplinary Design Optimization (MDO), and in turn attempts to evaluate the challenges and possible solutions through the results of a student survey. Overall, the findings suggest that a moderately guided project-based learning seems to be the most suitable alternative for teaching MDO in higher education. The learning activities can take place in the form of tutoring or supervision, while in addition to this, the learning experience can be further complemented with lectures, seminars, and tutorials.

The general course evaluation shows that the efforts of the authors have had a positive impact on the teaching of MDO. More specifically, the students appreciated the fact that they got to work with realistic case studies, and they were also impressed to discover the potential of MDO in terms of design space exploration. The development of high-fidelity models and the increased amount of simulation time were identified as the most critical challenges of the project, while as far as the MDO theory is concerned, it was found that students are often facing difficulties with concepts such as decomposition architectures, optimization algorithms, post-optimal analysis, and metamodels.

\section{REFERENCES}

Agte, J., De Weck, O., Sobieszczanski-Sobieski, J., Arendsen, P., Morris, A., and Spieck, M. (2010), "MDO: Assessment and direction for advancement-an opinion of one international group", Structural and Multidisciplinary Optimization, Vol. 40, No. 1-6, pp. 17-33.

Della Vecchia, P., Aigner, B., van Gent, I., Ciampa, P. D., and Prakasha, P. S. (2018), "Collaborative open source aircraft design framework for education-agile academy initiatives and results", International Council of the Aeronautical Sciences.

Gerber, D. J., and Flager, F. (2011), "Teaching design optioneering: a method for multidisciplinary design optimization”, Computing in Civil Engineering, pp. 883-890.

Giesing, J., and Barthelemy, J. F. (1998), “A summary of industry MDO applications and needs,” 7th AIAA/USAF/NASA/ISSMO Symposium on Multidisciplinary Analysis and Optimization.

Jasa, J. P., Hwang, J. T., and Martins, J. R. (2018), "Open-source coupled aerostructural optimization using Python”, Structural and Multidisciplinary Optimization, Vol. 57, No. 4, pp. 1815-1827.

Martins, J. R., and Lambe, A. B. (2013), "Multidisciplinary design optimization: a survey of architectures", AIAA journal, Vol. 51, No. 9, pp. 2049-2075.

Mason, W. H., Gürdal, Z., and Haftka, R. T. (1995), "Experience in multidisciplinary design education", ASEE Annual Conference.

Nicknam, M., Bernal, M., and Haymaker, J. (2013), “A Case Study in Teaching Construction of Building Design Spaces", 31 st Conference on Education and research in Computer Aided Architectural Design.

Papageorgiou, A., Tarkian, M., Amadori, K., and Ölvander, J. (2018), “ Multidisciplinary Design Optimization of Aerial Vehicles: A Review of Recent Advancements “, International Journal of Aerospace Engineering.

Simpson, T., and Martins, J. R. (2011), "MDO for complex engineered systems: Report from a national science foundation workshop", Journal of Mechanical Design, Transactions of the ASME, Vol. 133, No. 10.

Viana, F. A. C., Simpson, T., Balabanov, V., and Toropov, V. (2014), "Metamodeling in multidisciplinary design optimization: how far have we really come?", AIAA Journal, Vol. 52, No. 4, pp. 670-690. 